\section{Topical anesthesia of the airway using fibreoptic bronchoscope and the MADgic $\AA$ atomizer in patients with predicted difficult intubation}

To the Editor:

Whenever difficulty in ventilation and laryngoscopic intubation is anticipated, awake fibreoptic intubation is often the safest option. ${ }^{1}$ However, effective local anesthesia of the airway is mandatory for patient comfort and subsequent successful instrumentation. ${ }^{2}$ The "spray as you go" technique using fibreoptic bronchoscope (FOB) provides flexibility in selectively anesthetizing respiratory passages, but cannot provide topical anesthesia as effectively as transtracheal injection of lidocaine. ${ }^{3}$ This may be related to the fact that local anesthetic injected by the working channel of FOB or epidural catheter cannot be reduced to extremely fine cloud-like particles. The MADgic ${ }^{\circledR}$ atomizer (Wolfe Tory Medical Inc., Salt Lake City, UT, USA) is a new device that has the ability to provide atomized topical solution directly to the airway mucosa. ${ }^{4}$ One of its disadvantages is the requirement for direct laryngoscopy to provide access to the pharynx, larynx and trachea, which may cause airway stimulation that the awake patient cannot well tolerate. In contrast, exposure of the airway structures using a FOB can attenuate or even avoid direct airway stimuli. We have successfully combined a FOB (Olympus LF-DP or LF-TP, Tokyo, Japan) and a MADgic ${ }^{\circledR}$ atomizer to provide topical anesthesia of the hypopharynx, larynx and trachea before tracheal intubation.

This combined technique requires an elastic 5 $\mathrm{mm}$ lactoprene tube $3 \mathrm{~cm}$ in length. The lumen of the lactoprene tube is well lubricated using a watersoluble lubricant. Both the distal bending section of the FOB and the applicator portion of the MADgic ${ }^{\circledR}$ atomizer are inserted into the lactoprene tube until their tips protrude just beyond the distal end of lactoprene tube (Figure). With local Ethics Committee approval, 15 patients scheduled for elective surgery who had a predicted difficult intubation gave written informed consent to participate in a clinical evaluation of this combined technique. Of the 15 patients, three had limited mouth opening (inter-incisor distance of $18-22 \mathrm{~mm}), 11$ had limited extension of the head, and one patient had micrognathia (thyromental distance of $4.2 \mathrm{~cm}$ ).

Following topical anesthesia of the oropharynx with traditional spray techniques, fentanyl $1.5 \mu \mathrm{g} \cdot \mathrm{kg}^{-1}$ was administered intravenously and midazolam (dose

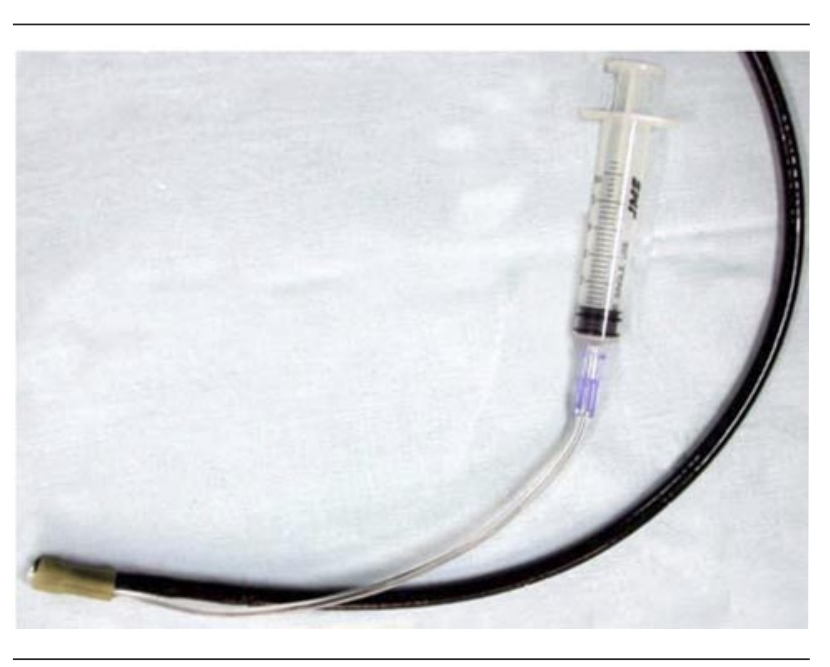

FIGURE The fibreoptic bronchoscope MADgic ${ }^{\circledR}$ unit. The fibreoptic bronchoscope and the applicator portion of MADgic ${ }^{\circledR}$ atomizer are assembled via a lactoprene tube.

range $1-8 \mathrm{mg} i v$ ) was titrated to the desired level of sedation, with the patient calm, falling asleep if undisturbed, but still able to respond to verbal commands or gentle stimulation. An airway intubator was inserted into the oropharynx, and a jaw thrust or gentle forward pull of the tongue was performed by an assistant. The operator introduced the FOBMADgic ${ }^{\circledR}$ unit through the airway intubator into the hypopharynx. By gentle rotation of the body of the FOB and manipulations of the tip control lever, the tip of the FOB-MADgic ${ }^{\circledR}$ unit was positioned near the bilateral piriform recess and epiglottic vallecula. Then the assistant used the MADgic ${ }^{\circledR}$ atomizer to spray $3 \mathrm{~mL}$ of $2 \%$ lidocaine in three aliquots onto these targeted areas. After three minutes, the FOBMADgic ${ }^{\circledR}$ unit was again inserted and positioned above the vocal cords. Two percent lidocaine $(1 \mathrm{~mL})$ was sprayed onto the vocal cords. This procedure was repeated two to three times until adequate anesthesia of the vocal cords was observed, as evidenced by cessation of the laryngeal response to further lidocaine administration. The FOB-MADgic ${ }^{\circledR}$ unit was then advanced into the trachea and its tip was positioned $2 \mathrm{~cm}$ below the vocal cords. Two percent lidocaine 2 $\mathrm{mL}$ was sprayed into trachea during inspiration. In five patients requiring nasotracheal intubation, the nasal mucosa of both nostrils was also prepared with lidocaine gel and vasoconstrictor before intubation. The fibreoptic intubation was started five minutes after the tracheal spray of lidocaine.

In all patients the FOB-MADgic ${ }^{\circledR}$ unit was successfully directed to the different targeted areas using one 
to two attempts. After the final spray, the mean visual analogue scores for pain, anxiety and coughing were $7.8 \pm 1.3,6.5 \pm 1.7$ and $5.2 \pm 1.5$, respectively (where 0 was described as awful and 10 as enjoyable). All subjects reported the airway spray of lidocaine to have been acceptable; two found it enjoyable. The time from start to final spray of lidocaine using the FOBMADgic ${ }^{\circledR}$ unit ranged from 21 to 28 min. The median dose of lidocaine was $2.5 \mathrm{mg} \cdot \mathrm{kg}^{-1}$ (range $2.1-2.8$ $\left.\mathrm{mg} \cdot \mathrm{kg}^{-1}\right)$. No patient experienced any local anesthetic side-effect and fibreoptic intubation was successfully completed on the first attempt in all patients. During the procedure, patient reaction scores $($ no reaction $=$ 1 , slight grimacing $=2$, strong grimacing $=3$, verbal objection $=4$, defensive movement of the head, hands or feet $=5)^{5}$ were $1.9 \pm 0.6$ with nasotracheal intubation and $1.6 \pm 0.7$ with orotracheal intubation, respectively.

According to this preliminary experience, we consider that using a combination of FOB and MADgic ${ }^{\circledR}$ atomizer for airway topical anesthesia has several advantages. First, it can provide excellent topical anesthesia of the airway. Second, this technique is well tolerated by the awake sedated patient. Third, it is non-invasive and therefore has minimal risk of airway damage. Fourth, there is no restriction on the patients' head and neck position. However, it must be pointed out that a limitation of this technique is its inability to anesthetize the nasal mucosa. To reduce airway damage caused by repeated manipulation, the FOB-MADgic ${ }^{\circledR}$ unit should not be inserted through the nasal cavity, because it has an external diameter of about $6-8 \mathrm{~mm}$.

Fu S. Xue MD

Quan Y. Yang MD

$\mathrm{Xu}$ Liao MD

Jian H. Liu MD

Shi Y. Tong MD

Plastic Surgery Hospital, Chinese Academy of Medical Sciences and Peking Union Medical College, Beijing, People's Republic of China.

E-mail: fruitxue@yahoo.com.cn

Accepted for publication August 8, 2007.

\section{References}

1 Kundra P, Kutralam S, Ravishankar M. Local anaesthesia for awake fibreoptic nasotracheal intubation. Acta Anaesthesiol Scand 2000; 44: 511-6.

2 Simmons ST, Schleich AR Airway regional anesthesia for awake fiberoptic intubation. Reg Anesth Pain Med 2002; 27: 180-92.

3 Graham DR, Hay JG, Clague, Nisar M, Earis JA.
Comparison of three different methods used to achieve local anesthesia for fiberoptic bronchoscopy. Chest 1992; 102: 704-7.

4 Supbornsug R, Osborn IA. Topicalization of the airway using the glidescope. Anesth Analg 2004; 99: 1263-4.

5 Puchner W, Egger P, Pubringer F, Lockinger A, Obwegeser J, Gombotz H. Evaluation of remifentanil as single drug for awake fiberoptic intubation. Acta Anaesthesiol Scand 2002; 46: 350-4.

\section{Ultrasound-guided continuous sciatic nerve blocks in two children with venous malformations in the lower limb}

\section{To the Editor:}

We write to share our recent clinical experience with ultrasonographic-guided continuous sciatic nerve blocks in two children. The first was a three-year-old girl, weighing $30 \mathrm{~kg}$, who had Proteus-like syndrome with serious congenital venous malformations extending from her left lower back via the buttock into the left foot. She was scheduled for a Lisfranc midfoot amputation. For postoperative analgesia an ultrasoundguided sciatic nerve block was planned. In the prone position, using a linear 7-13 MHz ultrasound probe (Sonosite Micromaxx, Bothell, WA, USA), the slightly blurred sciatic nerve surrounded by venous structures was visualized (Figure, panel A). Under ultrasonographic control in a long-axis view, a $4 \mathrm{~cm}$ insulated needle was inserted on the medial side at the distal $2 / 3$ of the thigh. Avoiding vascular structures the needle was brought into close proximity of the nerve and 5 $\mathrm{mL}$ ropivacaine $0.375 \%$ was injected, which spread circumferentially around the nerve. A $20-\mathrm{G}$ polyamide catheter was threaded through the needle, and the position verified by injecting $2 \mathrm{~mL}$ ropivacaine $0.375 \%$ under ultrasonographic control. Surgery proceeded uneventfully and no other analgesics were given. Postoperatively a perineural infusion of ropivacaine $0.2 \%$ at a rate of $1 \mathrm{~mL} \cdot \mathrm{hr}^{-1}$ was started for five days. During this period, no hematomas at the catheter insertion point were observed, nor did the child need additional pain relief. The parents and nurses judged the postoperative pain relief as excellent.

A second child $12 \mathrm{yr}$ of age, weighing $33 \mathrm{~kg}$, with severe congenital venous malformations in lower back, pelvis and right leg, was scheduled for revision of an amputation stump of the lower leg. One month earlier, a lower limb amputation had been performed because of pain and asymmetrical growth. We undertook an ultrasound-guided sciatic nerve block for 